\title{
Psicología y Didácticas: demarcación e interconexión
}

\author{
CÉSAR COLL
}

\author{
Universidad de Barcelona
}

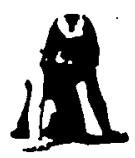

Como se señala en el artículo introductorio de esta monografía, la reunión que estuvo en el origen de los materiales que en ella se incluyen partía de unos supuestos y perseguía unos objetivos. El desarrollo de las sesiones de trabajo mantenidas a lo largo de los tres días que duró la reunión puso de manifiesto que los supuestos eran en buena medida acertados y los objetivos alcanzables, además de compartidos por la práctica totalidad de los asistentes. Didactas y psicólogos pudimos comprobar hasta qué punto somos a menudo desconocedores de nuestras aportaciones respectivas a una mejor comprensión de los procesos escolares de enseñanza y aprendizaje; y también que esta «fuerte y pertinaz tendencia» a ignorarnos mutuamente no encuentra justificación alguna ni en la naturaleza de los problemas que estudiamos ni en las finalidades que perseguimos.

Algunos de nosotros hemos tenido por primera vez la oportunidad de hablarnos y escucharnos directamente, de identificar puntos de acuerdo sustanciales y también discrepancias más o menos profundas. Pero sobre todo hemos tenido la experiencia inédita de intercambiar reflexiones y puntos de vista en un clima de respeto y aceptación de nuestros respectivos campos de trabajo y de nuestras tradiciones epistemológicas, conceptuales y metodológicas. Hemos aceptado y practicado escrupulosamente la regla propuesta de no operar con representaciones cruzadas en las que nos cuesta reconocernos a unos y otros y, en vez de partir de la representación que cada uno de los dos colectivos tiene del ámbito de trabajo del otro, hemos tratado de explicarnos mutuamente cuáles son nuestras preocupaciones básicas, nuestros objetivos y nuestros marcos de referencia.

La calidad de las ponencias, y muy especialmente la intensidad y la riqueza de las discusiones que han generado - recogidas también sintéticamente en esta publicación - muestran el acierto y la bondad de esta manera de proceder y ponen de relieve las enormes ventajas que pueden derivarse de un diálogo fluido

Correspondencia con autor: Departamento de Psicología Evolutiva y de la Educación. Facultad de Psicología. División de Ciencias de la Salud. Universidad de Barcelona. C/ Adolf Florensa, s/n. 08028. Barcelona. 
y de una colaboración continuada entre, por una parte, la parcela de la psicología que se interesa por el aprendizaje y la enseñanza de contenidos escolares específicos y, por otra, las didácticas que se ocupan de la enseñanza y el aprendizaje de esos mismos contenidos.

Sería un contrasentido, habida cuenta de los objetivos y del desarrollo de las sesiones de trabajo, intentar establecer unas conclusiones. A menos unas conclusiones que fueran más allá de la convicción, ampliamente compartida por los participantes, de que es posible y conveniente incrementar las relaciones y la colaboración entre los respectivos ámbitos disciplinares. No se trata pues, con estas páginas, de forzar unas conclusiones que en ningún momento fueron formuladas como tales al término de la reunión. Mi objetivo es más bien retomar algunos argumentos expuestos en la ponencia introductoria y presentar, al hilo del resto de ponencias y de las correspondientes discusiones, unos breves comentarios y valoraciones personales.

\section{Las relaciones entre psicología y didácticas}

El espacio de problemas relativo al estudio de los procesos escolares de enseñanza y aprendizaje parece ser efectivamente un espacio en el que nos situamos por igual, y en consecuencia compartimos en buena medida, no sólo los didactas y al menos algunos psicólogos de la educación y de la instrucción, sino también otros especialistas y profesionales. Sin embargo, el hecho de compartir este espacio no supone en absoluto renunciar a lo que es específico y propio de cada uno de estos colectivos y de sus correspondientes ámbitos disciplinares. Para decirlo en términos positivos, psicólogos y didactas nos situamos de manera distinta en este espacio de problemas. Mi impresión, además, es que no sólo nos situamos de manera distinta, sino que queremos situamos de manera distinta: hay elementos específicos en ambos enfoques, el didáctico y el psicológico, y este hecho no es percibido como algo intrínsecamente negativo, sino más bien todo lo contrario, como algo que puede ser positivo y que, en cualquier caso, es algo en buena medida querido, buscado y asumido tanto por didactas como por psicólogos.

Sin embargo, y una vez aceptada la especificidad de ambos acercamientos como un hecho real -además de explícitamente asumido e incluso deseado-, conviene hacer dos matizaciones. En primer lugar, no es siempre fácil precisar concretamente en qué consiste dicha especificidad. La frontera entre la aproximación psicológica y la aproximación didáctica al estudio de los procesos escolares de enseñanza y aprendizaje es más bien sinuosa y en muchos tramos aparece considerablemente desdibujada. En segundo lugar, los objetivos que se persiguen, los problemas que se plantean, los procedimientos que se sugieren para abordarlos y los referentes teóricos desde los que se trabaja trascienden a menudo la frontera establecida, siendo aceptados o no por igual en ambos campos disciplinares. Respecto a algunas cuestiones clave, la línea divisoria o la contraposición de puntos de vista no pasa por la línea de demarcación disciplinar que separa la psicología de la didáctica, sino por otras coordenadas. En el seno de la psicología de la educación y de la instrucción, al igual que en el seno de las didácticas de los contenidos escolares, coexisten enfoques y explicaciones diversos y heterogéneos de los procesos de enseñanza y aprendizaje que se comparten o no sin que ello se relacione directamente con el hecho de situarse en uno u otro de los dos campos disciplinares. 
Así pues, la realidad de sendos acercamientos específicos desde la psicología y desde la didáctica al estudio de los procesos escolares de enseñanza y aprendizaje ha de completarse necesariamente, a mi juicio, con la constatación de la existencia de múltiples interconexiones e interrelaciones entre ellos. La utilización y apropiación mutuas, o simplemente compartidas, de finalidades, temáticas, enfoques, conceptos, categorías explicativas y procedimientos de indagación e intervención es quizás uno de los rasgos más llamativos y sobresalientes de las ponencias presentadas y, con toda seguridad, uno de los que mayor acuerdo han concitado en el transcurso de la reunión.

\section{Tendencias y movimientos compartidos en la construcción del objeto de estudio}

Como señalaba en la ponencia introductoria, pienso que estas interrelaciones e interconexiones van a incrementarse aún más si cabe en el futuro como consecuencia de una serie de tendencias y movimientos, presentes por igual en la actualidad en el campo de las didácticas y de la psicología, que introducen novedades importantes en la construcción del objeto de estudio, en la manera de definirlo, de acercarnos a él y de intentar explicarlo. Los materiales incluidos en esta monografía reflejan claramente tanto la realidad de dichas tendencias y movimientos como su vigencia en ambos campos disciplinares. Sin embargo, una lectura atenta de los mismos pone de relieve que, en la mayoría de los casos, las tendencias apuntadas son aún hoy por hoy más bien una serie de aspiraciones y de perspectivas de futuro que realidades plenamente asumidas. Las novedades a las que apuntan no son igualmente aceptadas e interpretadas por los colectivos de didactas y de psicólogos ni, profundizando un poco más en la misma dirección, por los diferentes sectores que configuran ambos colectivos.

Particularmente ilustrativa a este respecto es la tendencia que señalábamos hacia un modelo teorético de naturaleza constructivista de los procesos escolares de enseñanza y aprendizaje. El recurso a los principios constructivistas como referente teórico de investigaciones y propuestas ha sido efectivamente una constante en la reunión. Sin embargo, en el transcurso de las discusiones hemos podido comprobar que tras el término «constructivismo» se esconden interpretaciones y explicaciones diversas y no siempre coincidentes. En un momento determinado de las mismas, y simplificando quizás en exceso lo que ha sido sin lugar a dudas un debate mucho más rico y lleno de matices, me ha parecido detectar, por así decirlo, dos maneras distintas de apelar al constructivismo; o mejor aún, dos finalidades distintas en el recurso al constructivismo como referente teórico.

En ocasiones, el constructivismo se entiende fundamentalmente como un modelo explicativo de los procesos psicológicos subyacentes a los aprendizajes que alumnos y alumnas llevan a cabo en la escuela y, por tanto, susceptible de dar cuenta de los mismos. Como señala acertadamente Juan Ignacio Pozo en su ponencia al caracterizar la «investigación psicológica en torno al conocimiento científico y su adquisición», en este caso 


\section{0}

Además, y siempre en palabras de Juan Ignacio Pozo, en este caso

«el acercamiento de los procesos de aprendizaje/enseñanza suele ser más bien ocasional y secundario y sólo en la medida en que es necesario para alcanzar ese objetivo primordial».

En otras ocasiones, en cambio, el constructivismo se entiende fundamentalmente como un modelo explicativo, o mejor, como el intento de elaborar un modelo explicativo de los procesos de aprendizaje que son el resultado de procesos específicós de enseñanza y que debe contemplar, por tanto, no sólo la actividad constructiva del alumno - los procesos psicológicos subyacentes al aprendizaje-, sino también la incidencia de la enseñanza sobre dicha actividad constructiva. En este caso, como señala no menos acertadamente Ignasi Vila en su ponencia relativa al aprendizaje y la enseñanza de la lengua,

«aquello que es importante investigar se refiere al tipo de ayuda pedagógica y a cómo se realiza su ajuste en relación a un contenido particular de aprendizaje que permite construir un determinado conocimiento a un alumno concreto».

de ahí su propuesta de centrar los esfuerzos en el

«estudio de los mecanismos de influencia educativa que promueven el aprendizaje de los procedimientos implicados en el uso del lenguaje»,

esfuerzos que el autor sitúa por igual en el campo de la psicología educativa y de la didáctica de la lengua.

Como puede comprobarse, el recurso al constructivismo tiene una significación netamente distinta en ambos casos y conduce a enfoques y prioridades diferentes en la intervención psicopedagógica y en la investigación de los procesos escolares de enseñanza y aprendizaje. Sin entrar en el debate de fondo, lo que me parece importante destacar en el contexto de la problemática de las relaciones entre psicología y didácticas es que no debemos apresurarnos a identificar la primera manera de entender el constructivismo como propia y exclusiva del acercamiento psicológico, y la segunda como propia y exclusiva del acercamiento didáctico. Si aceptamos la regla de dejar hablar a los protagonistas y de renunciar a las interpretaciones cruzadas, podremos comprobar que en la actualidad hay didactas que utilizan el constructivismo como marco teórico de referencia en la primera de las dos acepciones mencionadas; y también psicólogos, corro el propio Ignasi Vila, que utilizan el constructivismo como marco teórico de referencia en la segunda de las acepciones.

\section{Problemas y retos compartidos}

Pero las interconexiones e interrelaciones entre psicología y didácticas no se manifiestan sólo en el hecho de que se compartan; cada vez con mayor amplitud, finalidades, temáticas, enfoques, conceptos, categorías explicativas y procedimientos de indagación e intervención. Se comparten también problemas y dificultades de índole diversa. Una de las aportaciones más interesantes de la reunión ha sido precisamente, a mi juicio, que nos ha permitido identificar y formular un conjunto de problemas relativos al análisis, la comprensión y la planificación de los procesos escolares de enseñanza y aprendizaje. Permítaseme mencionar, a título puramente ilustrativo, algunos de estos problemas que constituyen otros tantos retos para todos aquellos que trabajamos en este campo, y ello con idependencia de que lo hagamos desde las didácticas o desde la psicología. 
(i) Las finalidades de la acción educativa y su incidencia sobre la forma, desarrollo y resultados de los procesos escolares de enseñanza y aprendizaje.

(ii) Las dimensiones social, institucional y relacional de los procesos escolares de enseñanza y aprendizaje y su toma en consideración efectiva, y no sólo retórica, en el análisis y la planificación de los mismos.

(iii) La naturaleza y características de la estructura cognitiva de los alumnos y alumnas, su dinámica interna y los mecanismos de cambio conceptual.

(iv) Los procedimientos de ajuste de la ayuda pedagógica y los mecanismos de influencia educativa susceptibles de inducir y orientar los cambios en la estructura cognitiva de los alumnos y alumnas. La importancia y el papel del conflicto cognitivo y sociocognitivo en este marco.

(v) La influencia del contexto y de la situación en los procesos de construcción del conocimiento sobre los contenidos escolares. En especial, la problemática relacionada con la tensión contextualización/descontextualización y sus implicaciones para la funcionalidad, generalización y transferencia de los aprendizajes escolares.

(vi) La conveniencia de disponer de visiones globales, integrativas y coherentes de los procesos escolares de enseñanza y aprendizaje que guíen y orienten la práctica docente y la actividad de otros profesionales de la educación.

Este último punto merece tal vez un breve comentario por cuanto introduce una problemática que, a tenor de la lectura de las ponencias y de las discusiones mantenidas durante las sesiones de trabajo, podría dar la impresión de que no presenta el mimo grado o nivel de urgencia y de interés para psicólogos y didactas. La conveniencia de disponer de una visión global, integrativa y coherente de los procesos escolares de enseñanza y aprendizaje ha sido especialmente, aunque no exclusivamente, subrayada por los didactas. Así, por ejemplo, García Armendáriz, Azcárate y Deulofeu, en su ponencia sobre Didáctica de las Matemáticas y Psicología, señalan que

«una de las cuestiones fundamentales que se plantean actualmente es la determinación de un marco teórico global que permita relacionar las distintas estructuras o jerarquías cognitivas locales referentes a contenidos específicos (como por ejemplo las estructuras aditivas, los conceptos de función, de derivada, de probabilidad...).»

Observaciones similares, aunque con diferentes matices, se encuentran prácticamente en todas las ponencias presentadas por los didactas y también en las aportaciones realizadas en el transcurso de las discusiones. La sintesis de la discusión de las ponencias sobre Psicología y Didáctica de las Ciencias de la Naturale$z a$ realizada por $\mathrm{M}$. Benlloch recoge nítidamente esta preocupación y arroja alguna luz sobre el origen de la misma:

«En la discusión quedó claro que, si bien la práctica en el aula puede alimentarse saludablemente de la información procedente de las investigaciones (...), éstas no alcanzan siempre a poder responder con la premura requerida (...). Se propuso que, mientras la líneas de investigación abiertas sigan tratando de establecer más claridad conceptual sobre los temas que preocupan, se buscará alguna teoría privisional... modesta... que permitiera a los profesores tomar decisiones urgentes, y que se investigara sobre ella. Con esta intervención se sugería que los investigadores deberían permitirse la licencia de elaborar soluciones más coyunturales, menos definitivas y más simples, pero más eficaces para mejorar el trabajo en el aula.»

En realidad, la diferencia de énfasis con que se planteó esta problemática tampoco se corresponde nítidamente con la línea de demarcación entre el colec- 
tivo de didactas y el colectivo de psicólogos. A mi entender, y como traduce la anotación realizada por Montse Benlloch, tiene que ver más bien con las relaciones teoría/práctica y, en especial, con las relaciones investigación/práctica profesional. Desde la perspectiva de la investigación, los intentos de elaborar una aproximación global, integrativa y coherente a los procesos escolares de enseñanza y aprendizaje son vistos con desinterés e incluso, en ocasiones, con manifiesta desconfianza. En cambio, desde la perspectiva de la práctica profesional - docencia, asesoramiento psicopedagógico, asesoramiento didáctico o curricular, etc. - adquieren una enorme importancia y se consideran convenientes, e incluso necesarios, para guiar, orientar y dotar de coherencia las actuaciones e intervenciones puntuales. Como señala Isabel Solé en la síntesis de la discusión de las ponencias sobre Psicología y Didáctica de la Lengua,

«desde ambas ponencias se ha señalado la necesidad, en el momento actual caracterizado por la existencia de múltiples respuestas parciales, de disponer a la vez de un marco global que oriente sobre la enseñanza en la escuela, un poco quizá al modo en que Daniel Gil reclamaba en una sesión anterior (...) "un marco general que de sentido a todas las cosas", como creí entender. Las aportaciones de los participantes en la discusión se han mostrado de acuerdo con esa necesidad, y han puesto de manifiesto la existencia de opiniones divergentes acerca de si el modelo que nos hace falta es un modelo general de los procesos de enseñanza y aprendizaje escolares o bien un modelo circunscrito al ámbito de la lengua».

Como señala Juan Ignacio Pozo en su ponencia, sería, pues, aconsejable, para seguir profundizando en el análisis de las relaciones entre psicología y didácticas, precisar el ámbito o nivel en el que nos situamos: el de la práctica docente, el de la formación de los docentes y de los otros profesionales o el de la investigación. Mi impresión, sin embargo, es que, una vez más, en lo que concierne a la discusión de si conviene o no perseverar en los intentos de elaborar un marco global de referencia para el análisis y la planificación de los procesos escolares de enseñanza y aprendizaje, las respuestas serán de uno u otro signo no tanto en función de que nuestro pronunciamiento se realice desde las didácticas o desde la psicología, sino más bien en función de que se realice desde una u otra opción epistemológica sobre las relaciones teoria/práctica.

\section{Sugerencias para impulsar el diálogo y la colaboración}

Entre los objetivos del encuentro figuraba en un lugar destacado el relativo a formular propuestas concretas dirigidas a impulsar un diálogo más fructífero y una colaboración más estrecha entre ambos colectivos. Los materiales incluidos en esta monografía dan fe de los esfuerzos realizados por los asistentes para alcanzar dicho objetivo. Las ponencias y las síntesis de las discusiones realizadas a partir de ellas contienen múltiples e interesantes propuestas a este respecto y sería redundante proceder aquí a enumerarlas de nuevo. No obstante, en ocasiones, cuando la importancia y el interés del mensaje lo requiere, la redundancia está justificada. Como estoy firmemente convencido de que ésta es una de esas ocasiones, quiero terminar repitiendo algunas de las propuestas formuladas:

(i) Organizar foros de encuentro y de discusión entre psicólogos y didactas que trabajan en un mismo ámbito, área o dominio de contenidos escolares.

(ii) Esforzarse, desde uno y otro colectivos, por conocer más las aportaciones respectivas al estudio de los procesos escolares de enseñanza y aprendizaje; 


\section{3}

utilizar las publicaciones didácticas como fuente de información habitual de los psicólogos y recíprocamente.

(iii) Potenciar la publicación de trabajos realizados desde la didáctica en revistas especializadas de psicología y recíprocamente.

(iv) Renunciar a la utilización de representaciones cruzadas: que cada colectivo defina cómo se sitúa en este espacio de problemas relativo a los procesos escolares de enseñanza y aprendizaje y renuncie a definir y valorar cómo debe situarse el otro.

(v) Renunciar a la aceptación acrítica, pero también al rechazo apriorístico, de las aprotaciones que tienen su origen en el otro ámbito disciplinar.

(vi) Constituir equipos de investigación integrados por psicólogos y didactas que aborden conjunta y colaborativamente, aportando sus respectivos puntos de vista, una misma problemática.

La puesta en práctica de estas y otras propuestas sólo depende, en último término, de nuestra convicción y de nuestros esfuerzos. Mi impresión personal es que, al menos en lo que concierne a los participantes en el encuentro, ambas condiciones se cumplían con creces. 